\title{
LA NEUTRALIDAD EN LA RED COMO POSIBILIDAD DE SIMETRÍA EN EL CONTROL CIUDADANO DE LA INNOVACIÓN CIENTÍFICA ${ }^{1}$
}

\author{
THE NET NEUTRALITY AS A POSSIBILITY OF SYMMETRY IN CITIZEN \\ CONTROL OF SCIENTIFIC INNOVATION
}

Helena Nadal Sánchez

\begin{abstract}
Internet ha cambiado fundamentalmente la realidad práctica y económica de la distribución del conocimiento científico y el patrimonio cultural. Por primera vez en la Historia, Internet ofrece la oportunidad de construir una representación global e interactiva del conocimiento humano, incluyendo el patrimonio cultural y la garantía universal de acceso. Nosotros, los abajo firmantes, nos sentimos obligados a afrontar los retos que Internet, como medio emergente y funcional para la distribución del conocimiento. Obviamente, este desarrollo modificará significativamente la naturaleza de la edición científica y el sistema existente de evaluación de la calidad.
\end{abstract}

Prefacio de la Berlin Declaration on Open Access to Knowledge in the Sciences and Humanities.

RESUMEN - Con la posibilidad de que los Proveedores de Servicios de Internet discriminen los accesos la red mediante un nuevo sistema de cobro en función de los servicios que el usuario contrate, desaparece la neutralidad de la red. Augurar todas las implicaciones de este hecho es imposible, pero si es lícito aventurar algunas consecuencias inmediatas para el campo de la innovación y de la investigación. Los principales puntos de reflexión que articulan este trabajo son: la relación entre capitalismo y restricciones de contenidos en la Red, el fin de la participación ciudadana en nuestras sociedades, el bloqueo del intercambio intelectual como freno en el desarrollo de la lógica de la innovación, la incapacidad de los seres humanos para influir en la configuración del conocimiento, la conversión del saber en objeto de mercado y el atentado contra el derecho a escoger el propio camino de desarrollo científico o intelectual. Finalmente se destaca el hecho de que el debate por la gestión de la red pone de manifiesto nuevamente las contradicciones entre los valores de la democracia y la legitimación del crecimiento económico ilimitado.

PALABRAS CLAVE - Neutralidad, acceso a la red, democracia, innovación, universal.

\footnotetext{
${ }^{1}$ Versión ampliada de la comunicación "Sin neutralidad en la red ¿Dónde situar la lógica de la innovación?" presentada en el VII Congreso Internacional Internet, Derecho y Política, Universitat Oberta de Catalunya, Barcelona, 11-12 de julio de 2011.

${ }^{2}$ Doctoranda del Departamento de Derecho Público de la Universidad de Burgos. E-mail: hnadalsanchez@hlna.org.
} 
ABSTRACT - If Internet Service Providers begin to discriminate against different kinds of websites and services through a new sistema of payment depending on the services that the consumer contracts, net neutrality will disappear. To imagine all of the implications of this act would be imposible, but it is possible to anticipate some immediate consecuences in the field of innovation and investigation. The principle themes of this article are: the relationship between Capitalism and Internet content restrictions, the end of citizen participation in our societies, the impiding of intellectual exchange such as blocking the development of the process of innovation, the inability of humans to influence the way of knowledge and the conversion of this knowledge into merchandise, and finally the attack on the right to choose one's own scientifical and intelectual path. Finally it is stressed that the battle for the management of the net brings to light again the contradictions between the values of democracy and the legitimization of unlimited economic growth.

KEYWORDS - Neutrality. Access to the net. Democracy. Innovation. Universal.

\section{INTRODUCCIÓN}

Por primera vez el ser humano puede afirmar con rotundidad que la realidad está desdoblada. Más allá de trasfondos metafísicos o religiosos que pueden dotar de sentido a la existencia, hoy en día no es aventurado decir que el ciberespacio se ha constituido como realidad alternativa y para muchos fundante de nuestras sociedades actuales. El modo como ha condicionado Internet no sólo a las culturas desde las cuales se tiene acceso a esta red sino indirectamente al resto del planeta justifica que cada cambio en ella sea observado y analizado con especial atención.

Internet como red paradigmática nos pone de nuevo ante el desafío de cómo regular los ámbitos humanos. Se constituye así como un campo de observación que permite estudiar las progresivas necesidades de un espacio sin fronteras, que traspasa el poder de los diferentes gobiernos. En este sentido, la red no sólo está transformando nuestras sociedades sino también nuestras concepciones del derecho, de la economía y de la investigación.

El objetivo de este trabajo, sin embargo, no se centra en analizar las posibilidades jurídicas respecto a los modos de regulación de Internet sino en intentar vislumbrar las supuestas implicaciones que tiene para las áreas de la investigación y de la innovación tanto el regular la red como el modo de hacerlo. 
La importancia de Internet para el progreso y difusión del conocimiento es clave. Hasta no hace mucho estas actividades eran propias de diversas élites políticas, teológicas, académicas o científicas según el momento histórico y que a su vez ejercían el control de su desarrollo. La asimetría que esto implicaba hacía que el resto de la población haya asumido un papel de receptor pasivo del conocimiento en el mejor de los casos o que haya sido condenado a la absoluta ignorancia en el peor de ellos.

Con el acceso generalizado a la red en la era de la revolución digital aparece, propiamente, la primera forma de simetría en el progreso y difusión del conocimiento y con ella las posibilidades de reales de universalización del mismo.

Si bien la consecuencia inmediata de la misma es la democratización del saber, en realidad el potencial de Internet se nos escapa de las mentes. La revolución digital, al igual que ya ocurriera con otras, como la revolución industrial, deja a los hombres de su tiempo la incógnita de las consecuencias que traerá ese nuevo giro en la historia humana. Por lo que respecta a la investigación y a la innovación Internet ha supuesto principalmente el acceso fácil a la documentación y como consecuencia la globalización del conocimiento.

Sin embargo, tras la pretendida necesidad de regulación de la red, como corresponde al resto de las parcelas humanas defendida por cada vez más gobiernos, se esconde la posibilidad de que dicha regulación facilite a los propios Proveedores de Servicios de Internet (ISP) tanto actuar al servicio de los intereses estatales colaborando en el control efectivo de los accesos a la red como la posibilidad de que establezcan servicios de pago discriminados según los contenidos que desee contratar el ciudadano. Tanto la primera como la segunda de las posibilidades se revelan problemáticas; en el primer caso, estos Proveedores traspasarían su carácter privado al adquirir competencias de carácter policial; en el segundo, del cual se ocupa preferentemente este trabajo, se daría por terminada, apenas conseguida, la simetría en el desarrollo del conocimiento y en corolario, la conquista de la universalización del conocimiento, de la investigación y de la innovación. 
En orden al desarrollo del trabajo, el primer apartado está dedicado de un modo preliminar a esclarecer diferentes significados e implicaciones de lo que supone una red neutral; el segundo centra el análisis sobre las pretensiones de los Proveedores de Servicios de Internet de discriminar el acceso a los servicios de la Red mediante sistemas de pagos diferenciados en función de las prestaciones que contrate el usuario; en el tercero se reivindica el acceso a Internet en abierto como una consecución de las democracias que no debe verse retrocedida; en el apartado cuarto y final la reflexión aborda las consecuencias que tiene para el ámbito de la innovación y de la investigación la restricción del acceso a Internet. Cierra este estudio una conclusión dedicada a proponer otro modo de entender el crecimiento de la Red a favor tanto de las sociedades que la hacen posible, es decir: todas.

\section{DIFERENTES ASPECTOS DE LA NEUTRALIDAD DE LA RED}

La neutralidad en la red consiste en que la conexión a Internet incluya todas las páginas web y servicios por igual o dicho de un modo más genérico, una red neutral es la que permite la comunicación punto a punto sin alterar su contenido $^{3}$. Esto implica en líneas generales que la red de redes se ha convertido en una realidad autónoma que supera las fronteras estatales y que el usuario la entiende como un espacio privilegiado de libertades, tanto por lo que respecta al acceso a servicios como por lo que respecta a la difusión y proyección de sus propias producciones ideológicas o intelectuales. "Desde algunos puntos de vista, Internet es el espacio de la libertad. Un lugar exento de intervenciones públicas en el que los cibernautas disfrutan de un poder de acción ilimitado. Sobre todo para comunicar y expresarse, para desarrollar experiencias de investigación y culturales de cualquier tipo, trascendiendo o no las fronteras de los Estados. Esta libertad no sólo es inmensa sino que tiene muy difícil limitación" (Muñoz 2000: 151)

El debate sobre si es posible regular la red (tanto si lo es desde el punto de vista técnico como si lo es desde el punto de vista legal) y cómo debería hacerse es candente en nuestros días. Chile ha sido pionero en garantizar la

\footnotetext{
3 “Neutralidad de red". Wikipedia.
} 
neutralidad de la red y, desde la postura opuesta, diversos países como Estados Unidos, España o Francia están legislando contra dicha neutralidad. En el primer caso se entiende el espacio cibernético como un logro tecnológico que permite una completa libertad para los usuarios a excepción de lo que concierne a controles parentales que sí están contemplados por ley. En el segundo, se considera en líneas generales que la regulación de internet permitirá "satisfacer las necesidades de las generaciones presentes sin comprometer las posibilidades de las del futuro para atender sus propias necesidades"4.

En España ha entrado en vigor desde el día 6 de marzo de 2011 la Ley de Economía Sostenible, cuya Disposición final cuadragésimo primera (más conocida como 'Ley Sinde') es relativa a la regulación de webs y la protección de la propiedad intelectual. A favor de dicha ley ${ }^{5}$ se sitúan quienes argumentan que las descargas sin consentimiento del autor no son legales, que el canon es para compensar la copia privada y que es discutible que la 'Ley Sinde' pisotee derechos fundamentales, ya que finalmente se ha incorporado la participación de un juez en diferentes momentos del procedimiento estableciendo un sistema con ciertas garantías (Gavilán: 2011). Joan Navarro (2011) hace notar que 'Quienes abogan por la 'libertad' y la 'gratuidad' en la Red, en realidad defienden el lucrativo negocio de quienes no pagan por los contenidos por los que intermedian" y añade "Es falso que exista un debate entre propiedad intelectual y libertad. Sin libertad no hay creación ni propiedad intelectual, y quien defiende los abusos no defiende la libertad, sino los privilegios (sean éstos tecnológicos o de casta)" para culminar exponiendo que "la ley Sinde no criminaliza a los usuarios de la Red, persigue a quien abusa, oculto tras la tecnología o el anonimato, del trabajo, curiosamente siempre intelectual, de otros". En contra de la mencionada ley ${ }^{6}$ se sitúan quienes consideran que la neutralidad de la red es imprescindible en el acceso, difusión y expresión de la cultura y que su eliminación sólo estaría favoreciendo a los intermediarios que se enriquecen a costa de hacerse con los derechos de los creadores. Añaden

\footnotetext{
${ }^{4}$ Ley 2/2011, de 4 de marzo, de Economía Sostenible. Artículo 2. Economía sostenible.

${ }^{5}$ En el marco político votaron a favor de la 'ley Sinde': PSOE, PP y CIU.

${ }^{6}$ En el marco político votaron en contra de la 'ley Sinde': IU-ICV-EUiA, UpyD, PNV, BNG y Coalición Canaria.
} 
que en este sentido la neutralidad de la red, que impulse las alternativas al 'Copyright' como las licencias 'Creative Commons' como base de una economía libre de injerencias y por tanto más moderna y productiva.

Manuel Castells describe el problema relativo al control de Internet ${ }^{7}$ como una transformación tecnológica y cultural ante la cual:

\begin{abstract}
Los detentores del poder de controlar la información a lo largo de la historia, es decir, los estados y las iglesias, reaccionaron con preocupación y, en los estados no democráticos, con hostilidad, tratando de restablecer el control administrativo de la expresión y la comunicación. Pero la ejecución del proyecto estatista sobre Internet se encuentra con obstáculos considerables. En los países democráticos, Internet se consolida como instrumento esencial de expresión, información y comunicación horizontal entre los ciudadanos y recibe la protección constitucional de las libertades. En todos los países, menos en las teocracias, la importancia económica y tecnológica de Internet, excluye que se pueda ignorar o relegar su amplio uso en la sociedad. Más aún, la ideología de progreso mediante la tecnología hace de la promoción de Internet un valor legitimador para gobiernos que fundan su estrategia en el desarrollo económico dentro del marco de la globalización. De ahí el complicado encaje de bolillos político entre la libertad y el control por parte de los Estados. (Castells 2001:1).
\end{abstract}

Por el contrario, frente a la importancia concedida por Castells a Internet en la cohesión social, Jürgen Habermas ${ }^{8}$ argumenta que:

\begin{abstract}
El Internet genera una fuerza centrífuga. Suelta una ola anárquica de circuitos de comunicación altamente fragmentados que de manera infrecuente se traslapan. Claro, la naturaleza espontánea e igualitaria de la comunicación ilimitada puede tener efectos subversivos en regímenes autoritarios. Pero la red en sí no produce esfera política alguna. Su estructura no está hecha para enfocar la atención de un público disperso de ciudadanos que forman opiniones de manera simultánea en los mismos temas y contribuciones que han sido escrutados y filtrados por expertos.
\end{abstract}

Habermas (1962) defiende la publicidad crítica en la nueva sociedad de masas más allá de los problemas que ésta pueda presentar, ya que ésta es la única solución viable para el proceso de comunicación pública que debe estar a la base de los compromisos políticos de la democracia. La publicidad crítica es entendida como expresión real de los conflictos y como vía de resolución de

\footnotetext{
${ }^{7}$ Manuel Castells (2009) trata exhaustivamente los problemas de la intervención en la esfera de las comunicaciones.

${ }^{8}$ Cfr. "Habermas y Castells, dos visiones de Internet". Para un análisis en detalle de la teoría de Habermas sobre Internet véase Froomkin (2003).
} 
éstos a través de la capacidad dialógica y la generación de consensos a partir de la voluntad común.

Como expone Fromkin (2003: 767), Habermas no afirma que las personas puedan resolver sus problemas simplemente sentándose alrededor de una mesa y hablando entre ellas, esta idea sería absurda e ingenua. Frente a esto reconoce que en la vida social buena parte de la interacción es de carácter estratégico y que la gente lleva en sus agendas muchos discursos preparados y tratan de ejercer su poder e influencia para conseguir lo que consideran como resultados ventajosos en lugar de ir buscando lo verdadero y lo justo ${ }^{9}$. Esta idea, en relación al tema de la plausibilidad de la cohesión social a la que da lugar la intercomunicación a través de internet revela la necesidad de apelar a intereses verdaderos y principios de honestidad en la construcción del diálogo social. La mera conexión entre comunicadores, por tanto, aunque tenga un alcance global, no implica un compromiso social con la democracia.

$Y$ es que, como toda construcción humana, también Internet está ligado no sólo a valores culturales o políticos, sino también a cuestiones económicas. $\mathrm{Si}$ el debate por la neutralidad de la red parece centrarse en la conveniencia de regulaciones como la 'ley Sinde' en España o la 'ley Hadopi' en Francia, existe otra faceta de la neutralidad de la Red cuestionada no ya desde los gobiernos sino por la intención de los Proveedores de Servicios de Internet o ISPs de controlar qué servicios de Internet ofrecen a sus usuarios y establecer tarifas específicas por esos servicios. Respecto a esta intención de los ISP el desplaza la reflexión del desarrollo de la tecnología de la comunicación del plano político-jurídico al plano económico. Esto no quiere decir que sus implicaciones sean únicamente económicas, más bien al contrario, plantean un problema que deriva en múltiples efectos que afectan también al desarrollo de la innovación y de la investigación.

De este último fenómeno, es decir, de cómo la discriminación de contenidos y servicios por parte de los ISP impactan en la innovación, el progreso intelectual y el acceso universal a las Tecnologías de la Información y la Comunicación (TIC) se hace cargo este trabajo.

\footnotetext{
${ }^{9}$ Traducción propia del original.
} 


\section{LA DISCRIMINACIÓN DESDE LOS PROVEEDORES DE SERVICIOS DE INTERNET (ISP)}

En líneas generales se puede afirmar que disfrutamos de una red neutral, es decir, libre de restricciones en el contenido y servicios, por lo que respecta a cómo accedemos a ella a través de nuestros ISP.

Sin embargo los Proveedores de Servicios de Internet cada vez consideran mayores ventajas en la discriminación de diferentes clases de páginas web, plataformas y servicios. El país donde esta circunstancia ha cobrado mayor relieve es Estados Unidos, donde la Federal Comunications Commision (FCC) analiza la conveniencia de mantener Internet en abierto (o neutralidad de la red) o permitir a los ISP la discriminación en los accesos a la red. Pero también en Europa diversas compañías de telecomunicaciones han seguido la idea de las norteamericanas y han encargado a la consultora A. T. Kearney un estudio sobre modelos alternativos de acceso a Internet en los que se contempla la discriminación de servicios por parte de los ISP.

Actualmente el usuario todavía puede utilizar Internet tanto como quiera y como quiera por lo que respecta a los modos de acceso a la red; sin embargo, si los ISPs llegaran a controlar dicho acceso entonces podrían legalmente limitar las velocidades de diferentes páginas web y servicios (de hecho ya se está haciendo esto con descargas ilegales) y el cobrarían al usuario dependiendo del tipo de uso que contrate con su proveedor. Yendo más allá, esto podría suponer que cada ISP llegara a bloquear servicios que ofrecen otros proveedores, como por ejemplo, el correo electrónico, permitiendo únicamente el acceso al suyo propio.

Las razones que sostienen los ISP a favor de la discriminación están relacionadas con el congestionamiento de la red. Durante los años 1990, ante el aumento de demanda las mayores compañías de telecomunicaciones empezaron a proveer de un acceso privado a los usuarios y empezaron a cobrar por este servicio. Con el desarrollo exponencial de Internet, los ISP se vieron obligados a invertir cada vez más en la actualización de técnica e infraestructuras con el fin de abarcar el aumento de las necesidades de la Red. A día de hoy, los proveedores consideran que el modo de acceso a Internet no 
discriminado provoca un congestionamiento cada vez mayor y que este inconveniente lo está sufriendo el propio usuario.

Desde theopeninter.net (2010) se ofrece una guía sobre Internet en abierto de la que destacan una serie de razones a favor de no discriminación de proveedores; tales razones recogen el sentir de quienes están en contra de que el acceso a Internet sea selectivo: un acceso abierto a Internet es la gran tecnología unificadora de nuestra época y su control no debería estar a merced de las corporaciones; además un acceso abierto a Internet estimula la competencia, ayuda a prevenir injusticias en relación a los precios por su utilización, promueve la innovación, es más digno de confianza y más honesto, favorece los negocios y protege la libertad de conocimiento. Todas estas razones son suficientes para abogar a favor de la no discriminación de los ISP, pero además hay otras que se estima que se sitúan a la base del problema y de ellas específicamente trata este trabajo.

Por el contrario en el informe $A$ viable future for Internet, la consultora A. T. Kearney (2011: 1-3) explica cómo Internet, además de proporcionar importantes beneficios sociales sostiene una parte decisiva de la actividad económica y justifica su estudio sobre cómo puede continuar el crecimiento de la red de modo tal que siga asegurando los beneficios mencionados. La consultora sostiene que el tráfico reciente ha experimentado un crecimiento pronosticable a medio plazo pero impresionante en un futuro, lo cual implica desafíos serios respecto a la viabilidad del modelo actual de Internet para lo sucesivo.

Si bien es cierto que la tecnología todavía puede proporcionar una parte de la solución, incrementando la capacidad de las redes o utilizando nuevas técnicas de gestión del tráfico, destaca que estas medidas no son suficientes ya que éstas responden al actual modelo de Internet que conlleva problemas como la limitación de incentivos económicos para los ISPs (denominados "Over the Top") y que la inversión que se destina a dar soluciones de congestionamiento en realidad es débil.

Así que se proponen otras opciones posibles para nuevos modelos que puedan resolver los problemas descritos y asegurar, de una manera adecuada, el progresivo incremento de la red en beneficio de todos. 
La consultora propone cuatro opciones no excluyentes, que consisten en incrementar los precios al usuario con la probabilidad de aumentar el volumen de las descargas en el caso de que éste lo necesite, que los comerciantes paguen por el tráfico que genera su actividad, ofrecer mayor cantidad de servicios contratados directamente con el proveedor, como es el caso de los servicios Premium o aumentar servicios propios del proveedor que operen en paralelo con el actual modelo de Internet.

En cualquier caso, y ese es el dato esencial del informe de la consultora es la idea de que independientemente de las posibilidades que se elijan tanto la estructura como el tipo de facturación de los cargos debería evolucionar bajo las fuerzas de la competencia legal para alcanzar un equilibrio como ocurre en otros mercados bilaterales.

\section{REIVINDICANDO UN ACCESO EN ABIERTO}

Nuestra sociedad tecnológica se rige por tres principios: capitalismo, democracia y hedonismo moral. Los tres han dado consistencia a nuestra postmodernidad, nos han servido de referencia. Sin embargo, los problemas que plantea la neutralidad de la red rompen el equilibrio de la tríada y abren una escisión entre cada uno de ellos.

La idea de la consultora A. T. Kearney de identificar a Internet con un producto de mercado nos recuerda que nos regimos por los principios económicos de capitalismo. Su pretensión de que los pagos por el acceso a la Red se regulen por las leyes del mercado convierten a la "gran tecnología unificadora" en una propiedad, en una mercancía que queda incluida bajo el paradigma de que toda relación social debe regirse por relaciones de compraventa; aunque esto ponga en contradicción los aspectos cuantitativos y cualitativos del progreso humano.

Con la técnica bajo el capitalismo sucede lo mismo que con cualquier otro aspecto de la cultura actual: el capitalismo la ha hecho posible y la ha adulterado, ha abierto las puertas a la creatividad, al bienestar y a la belleza y ha procurado siempre someterlo todo a la ley del máximo beneficio. Por suerte o por desgracia, la tecnología como la pintura, la literatura, la religión o el 
deporte ni son ajenas al resto de las actividades sociales ni se limitan a reflejar mecánicamente las necesidades de otras partes de la estructura social. La tecnología actual es inseparable de la ciencia y de la industria, y estas es una de las principales actividades económicas. Pero estaríamos ciegos, si pretendiéramos reducir todos los problemas del desarrollo tecnológico y también todos lo valores o disvalores que en él se encierran a los problemas de la economía y a los valores o disvalores de la actividad económica. (Quintanilla 2005: 140)

Nos movemos además en la tesitura del paradigma teórico de la democracia liberal formal, que el Estado ordenaba la sociedad mediante reglas de no interferencia entre los individuos. "El objetivo del Estado democrático en este paradigma era, o bien proteger derechos de la persona que se consideraban universales y consustanciales a la naturaleza humana y, en consecuencia, anteriores al Estado, e inalienables -en la versión contractualista del liberalismo-, o bien al esfera de la acción de los individuos como seres sociales libres frente a la amenaza de sus semejantes o la arbitrariedad de los gobernantes -en la versión utilitarista del liberalismo-, o bien ambas cosas al mismo tiempo" (Caminal 2010: 211).

Ambas cosas a un tiempo es lo que asumen los ISP:

1. Por una parte entienden la propiedad como un derecho inherente a la condición humana y que todo es susceptible de ser poseído, incluso un posible bien social como Internet. Desde el paradigma neoliberal trascienden la idea inicial de sociedad mercantil como sociedad abierta. Locke puso límites a esa sociedad, "(...) al libre juego del uso privado de la propiedad. Se trata, claro está, de límites ideológicos, que no serían respetados en posteriores modelos liberales de inspiración lockeana. Locke creía en una moralidad individual, de corte agrarista, que determinaría los intercambios de mercado; creía que el hombre tiene deberes para consigo mismo, determinados por la ley de la naturaleza; consideraba la exigencia moral de poner límites a la apropiación privada (claúsula lockeana). En cambio los neoliberales contemporáneos no hablan de virtudes ni de deberes, desmoralizan el vínculo social, liberan el derecho a la propiedad de toda claúsula legitimadora" (Bermudo 2001: II-219). 
2. Por otra, entienden su pretensión como un derecho de todo ser libre que no debe verse amenazado por terceros.

Capitalismo y democracia en el sentido neoliberal del término sirven a los intereses de los ISP, pero la democracia también es el punto de partida de quienes defienden la no discriminación por parte de los proveedores. Entendida ahora como el gobierno que encarna la voluntad general, la protección de derechos y libertades se reconvierte en una exigencia de no comerciar con bienes públicos, dentro de los cuales se considera Internet, dicha protección "contiene los elementos sustentadores para la organización de la sociedad democrática y pluralista, defensora de expectativas de libertad de conciencia, de derechos humanos y de igualdad de todos los ciudadanos" (López 2009: 290).

Esta postura a favor de Internet en abierto es fruto no de una consideración arbitraria sino porque fue el TCP/IP fue desarrollado para una red neutral, sin restricción de contenidos y servicios, y si bien es cierto que progresivamente los padres de la Red de estos protocolos, Vint Cerf y Tim Beners-Lee, han ido estimando la conveniencia de una posible vigilancia o redireccionamiento, no han estimado oportuno la discriminación de los ISPs ${ }^{10}$, sino que consideran que los ISP deben tarificar en relación a la velocidad y no a la capacidad, de forma que así se defienda la neutralidad de la Red ${ }^{11}$.

Paralelamente ocurre que problemas como este son tratados por la opinión pública desde el predominante hedonismo moral al que nos tiene acostumbrada la sociedad tecnificada. Lejos de debatir las cuestiones desde supuestos ético-políticos entendemos que el placer es el modo de vida que satisface todos nuestros deseos; entendemos que la sociedad es un conjunto de individuos que buscan la gratificación personal que se suele sustentar en la adquisición constante de bienes. En este modelo, el consumo deja de ser causa de la competencia económica para serlo de la competencia psicológica entre individuos y la discriminación de los ISP deja de enfocarse como un asunto social que puede reducir beneficios comunes para considerarlo una afrenta contra una especie de regalo de la tecnología hecho para el hombre postmoderno que está al servicio de sus nuevas necesidades. La competencia

\footnotetext{
${ }^{10}$ Goldman (2005)

${ }^{11}$ Contreras (2009)
} 
psicológica se constituye dentro de una cultura de consumo masivo de bienes y posibilidades que hasta ahora se habían considerado inaccesibles. Este hecho hace que lo los significados de términos como 'derechos' y 'libertades' se acomoden a perspectivas hedonistas y pierdan la fuerza reivindicativa que han tenido a lo largo de su historia. De hecho, frecuentemente se identifica la legitimidad de nuestros gobernantes con su capacidad de mantener nuestro bienestar económico exclusivamente.

$Y$ sin embargo este es un problema crucial porque la diferencia tecnológica marca la diferencia social y el acceso discriminado a la Red redundará en un desigual progreso cultural, de la innovación y de la difusión de los logros alcanzados por esta última. El dominio de Internet trae consigo el dominio del conocimiento a nivel mundial; recuérdese que técnicamente, la red no posee fronteras. Si la tecnología nació con el objetivo de cubrir las necesidades planteadas por la sociedad, con la discriminación de proveedores habremos conseguido un paso más en la inversión de ese objetivo, en favor de que el propio medio genere más necesidades y con ello más desigualdades en el acceso a la información. Falta frecuentemente el recurso a los "derechos de participación social en el aseguramiento de un mínimo vital y en la defensa de la calidad de vida" (Belloso 1999: 209) que asegura el Estado Social de Derecho.

Únicamente el acceso a la información nos permite avanzar como sociedades porque es la base de la discusión abierta en torno a problemas, necesidades, implicaciones y alternativas. Sólo al discusión abierta fortalece la democracia y la libera del control económico y tecnocrático. En consecuencia, el acceso a la información es el primer principio democrático. Otra cuestión es si nuestras sociedades, asentadas en una moral hedonista están reivindicando dicho acceso siendo conscientes de que la información es la base para influir en la configuración de las sociedades y no un mero bien de satisfacción individual.

Yendo más allá pero en la misma línea es destacable el fenómeno de que se ha dado en denominar "multitudes inteligentes" y que en buena parte ha sido posible gracias al acceso universal a la red. Las multitudes inteligentes "son grupos de personas que emprenden movilizaciones colectivas -políticas, 
sociales, económicas- gracias a que un nuevo modo de comunicación posibilita otros modos de organización a una escala novedosa entre personas que hasta entonces no podían coordinar tales movimientos" (Rheingold 2004:13)

Pero el acceso a la información no es pilar únicamente de una sociedad democrática sino también un elemento fundamental en la lógica de la innovación científica e intelectual, que a su vez también es la base del progreso social y desde él es posible armonizar ciertos elementos del difusionismo y la invención independiente, posturas antropológicas en controversia que han intentado explicar el fenómeno de la invención en el ser humano:

\begin{abstract}
En esencia, el tema gira sobre la facultad de invención del hombre, cuando en algunos lugares del mundo, distantes entre sí encontramos artefactos 0 instituciones diferentes similares, 0 conceptos análogos ¿debemos resolver que todos ellos han sido inventados una sola vez y difundidos por las regiones en las cuales se los han encontrado, o bien podemos inferir que se originaron independientemente en esas diferentes regiones?

En lo que atañe a la mayor parte de las culturas, poca duda puede haber de que los elementos prestados predominan sobre los que han tenido su origen en el interior. El hombre, como se ha dicho es una criatura que encuentra más sencillo aprovecharse de alguna cosa que algún otro ha inventado que buscar él mismo las soluciones a sus problemas (Herskovits 2008: 540).
\end{abstract}

Tres son las palabras clave de este fragmento para la explicación de la lógica de la innovación y de la investigación en relación al acceso a Internet: "aprovecharse", "inventar" y "soluciones". En esta última confluyen las dos primeras; en una era en la que el conocimiento se constituye como piedra angular para la resolución de problemas y retos acuciantes de carácter global, la red permite un "aprovecharse" de lo ya construido para "inventar" o investigar a partir de ello y resolver también a un nivel global. Si se parte de que la copia es una de las bases de la creación y así parece que lo atestigua la antropología, el acceso universal a la red permite un avance rápido y en estructura fractal de las posibilidades de resolución de problemas. Nada hasta ahora, había provisto de semejante potencial a la especie humana.

Ya en el siglo XVIII las sociedades científicas se crearon con el fin de poner en común los trabajos de los científicos, entendiendo que con ello se favorecían futuras innovaciones, sino que, como es el caso de las Academias Inconformistas de Inglaterra, conectaron las investigaciones con el desarrollo industrial (Mason 2001-3: 32). Desde entonces la forma de evolucionar el 
conocimiento no ha cambiado en lo sustancial: la innovación necesita también del contacto entre investigadores, aunque contamos con ventajas sustanciales. Indudablemente la rapidez con la que nos suministrara información la red y la enorme cantidad de datos que nos proporciona han revolucionado la producción del conocimiento, pero sobre todo contamos con el potencial que tiene el ciberespacio a la hora de poner en contacto a los investigadores.

Si bien en los siglos de las sociedades científicas (XVIII y XIX) la ciencia y los avances en el conocimiento se podrían calificar de nacionales como atestigua Mason (2001-4: 59): "Durante el siglo dieciocho, los filósofos naturales de Francia y Gran Bretaña fueron los más importantes del mundo científico. (...) Sus actividades fueron complementarias, inclinándose los franceses hacia la interpretación teórica de la naturaleza y los ingleses, hacia la investigación empírica. (...) En las primeras décadas del siglo diecinueve los franceses estaban a la cabeza del mundo de la ciencia, pero no mantuvieron su impulso y para la década de los cincuenta y los sesenta los británicos se hallaban de nuevo a la cabeza. Con todo, la primacía británica no duró mucho, pues para finales de siglo Alemania había superado a Inglaterra y a Francia por lo que la ciencia se refiere"; hoy disponemos de la técnica para convertirlos en universales.

$\mathrm{Si}$ la conexión universal entre investigadores es esencial para el desarrollo del conocimiento también lo es la conexión entre todos los habitantes del planeta, "de hecho, Internet se convierte en la locomotora de digital que propicia el conocimiento exponencial de las publicaciones científicas en la Web y, en último término, del movimiento mismo del Open Access to Science o del Free Access to Science o Science Commons- que de todas estas maneras podría denominarse a un movimiento por otra parte bastante homogéneo- que, en resumidas cuentas, defiende y propaga el acceso gratuito al fruto de las investigaciones científicas" (Ortega, Rodríguez 2011: 90).

La idea de que el seno de las comunidades académicas es el principal foco de desarrollo y difusión del conocimiento queda superada por las múltiples evidencias de que al margen de ellas es posible trabajar a favor del progreso humano. La enciclopedia virtual Wikipedia y el sistema operativo Linux son ejemplos de ello. Ambas son iniciativas colaborativas basadas en la 
información compartida y por tanto muestras del fenómeno denominado "procomún", cuyos principales teóricos, entre otros, son Yochai Benkler ${ }^{12} y$ Elinor Ostrom ${ }^{13}$. Tanto una como la otra analizan cómo los bienes comunes obtenidos mediante el trabajo en red y las instituciones de acción colectiva, cuando son exitosos superan las condiciones de asimetría en las que se habían venido desarrollando las actividades humanas. Esta idea extrapolada a la neutralidad en la red supone que las aportaciones al conocimiento humano en forma de procomún, al permiten que cada vez un número mayor dentro de la población mundial "construya" información y que a su vez tenga un acceso en abierto y libre a la misma.

Es aquí donde nos entramos con las cuestiones relativas a las licencias libres de propiedad intelectual o copyleft, las cuales forman parte y son consecuencia de la universalización de las producciones intelectuales en su mayor parte difundidas en la red:

\begin{abstract}
De un estado analógico, de la escasez, que de alguna manera ha favorecido de alguna manera la individualidad genial y singularidad creativa del autor, se ha pasado a una etapa en la que lo colectivo cobra una nueva dimensión, como nueva concepción de la producción, donde un muchas ocasiones es difícil discernir quién es el autor. En este contexto, copyleft permite la reactivación de lo colectivo a través de la posibilidad de compartir más y mejores contenidos en más lugares, así como el enriquecimiento del procomún de los bienes culturales de los que todo el mundo puede aprovecharse. (Rodríguez 2008: 137,138)
\end{abstract}

Todo ello abre la posibilidad al control simétrico de la innovación científica por parte de la ciudadanía y contrapesa el hecho destacado por Lessig en el subtítulo de su obra Por una cultura libre (2011) de la utilización por los grandes grupos de comunicación de la tecnología y la ley para clausurar la creatividad.

\title{
5 CONSECUENCIAS PARA LA LÓGICA DE LA INNOVACIÓN
}

El bloqueo del intercambio intelectual que sobrevendría ante la explotación del ciberespacio por los ISP supondría un freno a la lógica de la

\footnotetext{
${ }^{12}$ Benkler, Y. (2006). The Wealth of Networks. How social production Transforms Markets and Production. New Haven: Yale University Press.

${ }^{13}$ Ostrom, E. (1990). El gobierno de los bienes comunes. La evolución de las instituciones de acción colectiva. México: Fondo de Cultura Económica. 2011 D. L.
} 
innovación, dentro del ámbito académico indudablemente, pero también a la innovación en general. Sólo en apariencia el conferir mayores ventajas a determinados servicios favorecería la especialización y el desarrollo del conocimiento. No se cuestiona que tener un acceso más rápido, más eficaz y más específico a determinadas instituciones o áreas de documentación como Universidades, Institutos de Investigación o publicaciones on-line implicaría a corto plazo mayores facilidades para el investigador siempre que pudiera acceder a ellas. Al fin y al cabo, estamos acostumbrados a pagar por el conocimiento y para realizar actividades de investigación. La cuestión es si es lo ideal y si esto no ha sido al fin y a la postre restrictivo para quienes no han podido acceder a estos medios. Si la red había venido a facilitar la generalización del conocimiento ¿por qué dar un paso atrás en esta cuestión?

Si se defiende la idea según la cual la innovación o la investigación deben quedar relegadas a una élite económica, eliminando el acceso universal a estos ámbitos es posible que no haya tenido en cuenta reflexiones del tipo de la que sostiene Cañas (2008: 13)

En la soledad de sus despachos o rodeados de las alabanzas y los
halagos de sus subordinados, los directores generales, los inversores
poderosos, los ingenieros imprescindibles en un proyecto o los
investigadores solicitados por todas las universidades pueden pensar
que dependen sólo de sí mismos, de sus méritos, de sus
conocimientos, de su valía, de su competencia, de su voluntad
inquebrantable... (...) Están equivocados. Desde antes de nacer y
hasta el preciso momento en que morimos, los humanos
dependemos de los demás en muchos sentidos, unos muy
manifiestos y otros más sutiles. Este es un hecho básico, crucial para
aplicar la razón a al conducta humana.

La innovación obedece o debe obedecer primordialmente a las demandas de la sociedad. Durante el siglo XIX la industria química alemana incrementó sus beneficios vinculando sus necesidades tecnológicas con la planificación científica. A partir de ese momento el saber empezó a conectarse con las necesidades reales, hecho que culminó durante la segunda mitad del siglo XX con la I+D (Innovación y Desarrollo) financiada por diversos gobiernos. La innovación pasó así de ser una actividad autónoma a estar al servicio de las necesidades estatales por las que era dirigida. Progresivamente este modelo de financiación estatal fue incluyendo a otras empresas productoras de tecnología y con ellas a la propia ciudadanía en la toma de decisiones. A la par 
se ha ido imponiendo el concepto capitalista de 'competencia' aplicado al ámbito de la innovación entendiendo ésta como no como la actividad destinada a resolver necesidades sociales sino a incrementar beneficios económicos.

Si el acceso abierto a la Red podría considerarse una innovación de carácter mayoritariamente social (entendiendo por tal que de ella se beneficiaba una gran parte de la sociedad), con el control de su acceso por parte de los proveedores de servicios de Internet, su desarrollo se incluiría en los modelos de innovación tecnológica al servicio de los beneficios de empresas privadas. Esto, que en nuestra sociedad es frecuente y no necesariamente perjudicial sí lo sería en este caso ya que los ISP estarían controlando el canal principal de divulgación del conocimiento.

Abundando en la cuestión y si se admite además la premisa que otorga primacía al contexto de descubrimiento científico según la cual existe una conexión entre condicionantes culturales y desarrollo del conocimiento y que por tanto la realidad está construida en respuesta a intereses determinados, aparece en cuestión la legitimidad de determinadas entidades para gestionar el acceso a las fuentes de información. Si fuera posible "medir la extraordinaria forma de realismo radical que la filosofía de la ciencia ha venido encubriendo" (Latour 2001: 16) entonces podemos concluir igualmente con Latour (2001: 23) que la razón no gobierna y que la fuerza triunfará finalmente, si entendemos por tal los intereses económicos en este caso de los ISP.

También es cierto que estas empresas de telecomunicaciones han asumido los costes financieros de la innovación necesaria para desarrollar estas estructuras que han permitido el crecimiento de Internet hasta convertirla en lo que hoy conocemos. Frente a la innovación básica, es decir, a largo plazo, que es la que suelen asumir los propios gobiernos, estas empresas desarrollan tecnologías más rentables a medio y corto plazo. Sin embargo, una cosa es invertir en el desarrollo de la Red y otra distinta es convertirla en objeto de su propiedad y someterla a las leyes del mercado. El impacto que esto provocaría en la sociedad, la política y la cultura es difícil de evaluar pero innegablemente provocaría la incapacidad de los ciudadanos para influir conscientemente en la configuración del conocimiento. 
De ahí a convertir el saber en objeto de mercado sólo hay un paso. De ello, al menos en el mundo académico vamos teniendo algunos indicios, ya antes de que se haya impuesto la discriminación de proveedores. El acceso a los principales bancos de datos es prohibitivo y por tanto discriminatorio para un usuario privado, que únicamente puede utilizar estos servicios formando parte de alguna de las instituciones que pueden financiar dichos accesos, como por ejemplo las universidades; en caso contrario el investigador en cuestión se encontrará en una situación de franca desventaja dentro de esta "cultura universitaria tecno-meritocrática, es decir, la cultura de la investigación por la investigación. (...) de la apertura de la investigación y de la idea de que lo más importante es la excelencia académica y la excelencia de la investigación" (Castells 2002: 4). La paradoja es que las propias productoras de innovación y conocimiento son las que posteriormente tienen que pagar por acceder a esas producciones; por eso, la publicación de trabajos de investigación y tesis doctorales en abierto y dentro de los repositorios de cada universidad es una medida adecuada para paliar el comercio del conocimiento.

Para resolver este problema diversas instituciones académicas apuestan por la producción y difusión del conocimiento en abierto, según esto:

La declaración de julio de 2008 del Fraunhofer Institut establece la
obligación taxativa de que todos sus miembros, todos los
investigadores que formen parte de sus diversos institutos, publiquen
en régimen de acceso abierto, bien dentro del repositorio institucional
- en forma de principio de preprint o de postprint, esencialmente, pero
no de manera exclusiva-, en revistas que estén plenamente acogidas
al reglamento de Open Access, o, como tercera posibilidad, en
cabeceras científicas cerradas, tradicionales, con un embargo
temporal en ningún caso superior a un año. (Ortega, Rodriguez 2011:
91)

Con todo ello no se está sosteniendo una visión utópica de Internet según la cual la Red "aporta un arrollador o imparable potencial para el desarrollo de las comunidades en vías de liberación, para el crecimiento exponencial del capital humano y social y para la realización de una plena participación democrática de las personas en toda decisión política", las bondades de Internet no han "erradicado de la humanidad el azote del odio, los prejuicios, la venganza, la pobreza ni la enfermedad, ni los erradicará" (Katz y Rice 2005: 15). Únicamente se está destacando el valor de mantener Internet 
en abierto como modo más universal de acceso al conocimiento del que actualmente disponemos.

\section{CONCLUSIONES}

Cada vez más las sociedades se adaptan a las exigencias de la técnica y no al contrario, como fue en un principio. Se puede decir que los parámetros del crecimiento económico han dejado de estar en pugna con los valores de la democracia para prácticamente sustituirlos y la innovación tecnológica llevada acabo por empresas privadas ha terminado prácticamente acaparando la técnica y la innovación tecnológica en su propio beneficio.

El debate sobre la gestión de la red plantea al usuario, pero también al ciudadano en general la oportunidad de recuperar los valores democráticos, esto es, de reivindicar intereses colectivos frente las pretensiones de los ISP. A pesar de que nuestra sociedad se define como 'de la información' o 'de la comunicación' en realidad esto no la convierte en una sociedad tan cohesionada como pudiera parecer, sus intereses se encuentran muy sectorizados y la esfera pública es débil, lo cual no debe confundirse con la tan destacada capacidad de convocatoria de la Red.

La verdadera democratización se da en la esfera pública y no en la sintonía de múltiples fragmentos sociales. Si los ISP discriminan los accesos a Internet están dividiendo aún más a la ciudadanía. Si bien es cierto que, a pesar de las innegables ventajas que ofrece la Red, ya se han constatado indicios de aislamiento entre usuarios habituales y si aquella queda sectorizada, este efecto podría multiplicarse, siendo así que no sólo el desarrollo del conocimiento sino la actividad de otras áreas humanas quedarían a merced de los intereses mercantiles de cada proveedor y el ciudadano se vería aún más descohesionado y con ello definitivamente más debilitado para hacer agruparse y hacer frente a cualquier amenaza o abuso de carácter global.

Los valores democráticos, pues, emergen cuando la gestión de la red deja de verse como el modo de aumentar sin límites las posibilidades de Internet, ya que es precisamente ahí, en la aparente necesidad de aumento sin 
límites de las prestaciones de la Red donde los ISPs encuentran la mayor justificación para imponer su idea de la discriminación de servicios. Ante un crecimiento ilimitado de la demanda de los usuarios, como está sucediendo en la actualidad, los proveedores encuentran al campo abonado para incrementar dichas prestaciones a cambio de asumir la Red como un valor de mercado aunque con ello acaben con uno de los grandes logros de Internet: hacer posible el acceso a la información desde prácticamente cualquier parte del mundo.

Ciertamente, este y otros logros de la Red de redes han sido posibles gracias a la inversión en infraestructuras que han venido realizando los propios proveedores, pero si como ha ocurrido con el desarrollo de casi todo avance tecnológico, nos acabamos encontrando con el dilema de si para aumentar el progreso y el bienestar, la propia especie humana deja de ser beneficiaria para ser pagana. Así las cosas parece más razonable debatir no tanto sobre si es legal y/o deseable que los ISP puedan hacerse dueños del acceso a la Red, sino más bien sobre si es posible someter el crecimiento de la Red a parámetros de desarrollo sostenible que la conviertan verdaderamente en un instrumento generalizado a favor del progreso humano y con un crecimiento más ajustado a las necesidades humanas y no tanto a las del mercado.

Si los proveedores de servicios de Internet logran finalmente discriminar el acceso a la que podría ser la principal fuente de información y comunicación, no quedará lugar entonces para la soberanía individual ni para la democrática al estar este fértil modo de acceso al conocimiento y a la realidad predeterminado por los intereses económicos de las grandes compañías.

\section{REFERÊNCIAS}

A. T. KEARNEY, Inc. (2010): A viable future model for the Internet. Chicago: Marketing \& Communications [disponible en línea en www.atkearney.com][Fecha de última consulta: 05/05/11].

BELLOSO, Nuria (1999). «Del Estado de Bienestar a la sociedad de Bienestar: la reconstrucción filosófico-política de su legitimidad» en BELLOSO, Nuria (coord.). Para que algo cambie en la teoría jurídica. Burgos: Universidad de Burgos. 
BENKLER, Y. (2006). The Wealth of Networks. How social production Transforms Markets and Production. New Haven: Yale University Press.

BERMUDO, José Manuel (2001): Filosofía política. Barcelona: Ediciones del Serbal. Vol. II.

CAMINAL, Miquel (1996): Manual de ciencia política. Madrid: Tecnos. D.L. 2010.

CERRILLO-I-MARTíNEZ, A., PEGUERA, M., PEÑA-LÓPEZ, I., VILASAU, M. (coords.) (2011): Actas del VIl Congreso Internacional Internet, Derecho y Política "Neutralidad de la red y otros retos para el futuro de Internet". Barcelona: Universitat Oberta de Catalunya. [Disponible en línea en http://edcp.uoc.edu/symposia/idp2011/proceedings/] [Fecha de última consulta: 15/01/2012]

CASTELLS, Manuel (2006). «Internet, libertad y sociedad: una perspectiva analítica". [Disponible en línea en http://www.uoc.edu/web/esp/launiversidad/inaugural01/intro_conc_imp.html]. [Fecha de última consulta: 20/04/11]. . (2002). «La dimensión cultural de Internet» [Disponible en línea

en http://www.uoc.edu/culturaxxi/esp/articles/castells0502/castells0502_imp.html]. Institut de cultura: Debates culturales. UOC [Fecha de última consulta: $11 / 05 / 11]$. . (2009). Comunicación y poder. Madrid: Alianza.

CONTERAS, Rufino (2009): «Vinton Cerf y Tim Beners-Lee abogan por la neutralidad de Internet» [Disponible en línea en http://www.computing.es/Noticias/200904210013/Vinton-Cerf-y-Tim-BernersLee-abogan-por-la-neutralidad-de-Internet.aspx]. Computing.es. [Fecha de última consulta: 10/05/11].

FROOMKIN, Michael A. (2003): «Habermas@discourse.net:Toward a critical theory of Cyberspace». Harvard Law Review. Vol. 116. January 2003. Nr. 3. [Disponible en línea en http://personal.law.miami.edu/ froomkin/discourse/ils.pdf] [Fecha de última consulta: 26/03/2012]

HABERMAS, J. (1962). Historia y crítica de la opinión pública. La transformación estructural de la vida pública. Barcelona: G. Gili. 1981 D. L.

GAVILÁN, Pablo (2011). "Yo estoy a favor de la ley Sinde». [Disponible en línea en http://pablogavilan.com/ 2011/02/yo-estoy-a-favor-de-la-ley-sinde/]. [Fecha de última consulta: 10/04/11]. 
GOLDMAN, Albert (2005). "Cerf Says Symmetry is Beautiful» [Dispohttp://www.isp-planet.com/news/2005/cerf-f2c.html.]. ISP-Planet. [Fecha de última consulta: 15/05/11].

HERSKOVITS, J. M. (1948). El hombre y sus obras. México: Fondo de Cultura Económica. 2011 D. L.

KATZ, James E., RICE, Ronald E. (2002). Consecuencias sociales del uso de Internet. Barcelona: Editorial UOC. D.L. 2005.

LATOUR, B. (1999). La esperanza de Pandora. Ensayos sobre la realidad de los estudios de la ciencia. Barcelona: Gedisa. 2011 D. L.

LESSIG, L. (2005). Por una cultura libre. Madrid: Traficantes de sueños. 2011 D. L.

LÓPEZ, Miguel A. (2009). «Laicismo día tras día. Para una fundamentación filosófico-política» en NUÑEZ, Paloma, ESPINOSA, Javier (coords.). Filosofía y política en el siglo XXI. Europa y el nuevo orden cosmopolita. Madrid: Akal.

MASON, Stephen E. (1985). Historia de las ciencias. Madrid: Alianza. D.L. 2001.

NAVARRO, Joan (2011). "A favor de Internet... y de la 'ley Sinde'». El País.com.

[http://elpais.com/artículo/opinión/favor/Internet/ley/sinde/elpepiopi/20110127_1 2/Tes?print=1] [Fecha de consulta: 30/03/11].

MUÑOZ, Santiago (2000). La regulación de red. Poder y Derecho en Internet. Madrid: Taurus.

ORTEGA, F., RODRÍQUEZ, J. (2011). El Potlatch digital. Madrid: Cátedra. Ostrom, E. (1990). El gobierno de los bienes comunes. La evolución de las instituciones de acción colectiva. México: Fondo de Cultura Económica. 2011 D. L.

QUINTANILLA, Miguel Ángel (1989). Tecnología: un enfoque filosófico y otros ensayos de filosofía de la tecnología. México: Fondo de Cultura Económica. D.L. 2005

RHEINGOLD, H. (2002). Multiudes inteligentes. Barcelona: Gedisa. 2004 D. L. RODRIGUEZ, N. (2008). "Producción artística y copyleft en el nuevo entorno digital" en LóPeZ, A., Ramírez, E. (coords.). Propiedad Intelectual. México: Centro Cultural de España, págs. 131-152.

«A Guide to the open Internet" (2010) [Disponible en línea en http://theopeninter.net]. [Fecha de última consulta: 01/05/11]. 
«Habermas y Castells: dos visiones de Internet» (2011). Nexos en línea [Disponible en línea en http://redaccion.nexos.com.nex/?p=1350]. [Fecha de última consulta: 19/05/11].

Ley 2/2011, de 4 de marzo, de Economía sostenible. BOE. Núm. 55. Sec. I. Pág. 25033.

«Neutralidad de red». Wikipedia. La enciclopedia libre. [http://es.wikipedia.org/wiki/Neutralidad_de_red] [Fecha de consulta: 01/04/11].

Professor Convidado 